we may

each have

'personalized'

epigenomic

signatures

that provide

information

about our risk

of disease.
COMPLEX DISEASE

\section{Epigenomics gets personal}

Despite much recent success in identifying variants that are associated with complex diseases, our ability to predict disease risk from genetic information remains limited in most cases. A recent study suggests a new approach to this problem: it shows that we may each have 'personalized' epigenomic signatures that provide information about our risk of disease.

To be useful for prediction, epigenomic patterns would need to be stable in individuals. However,

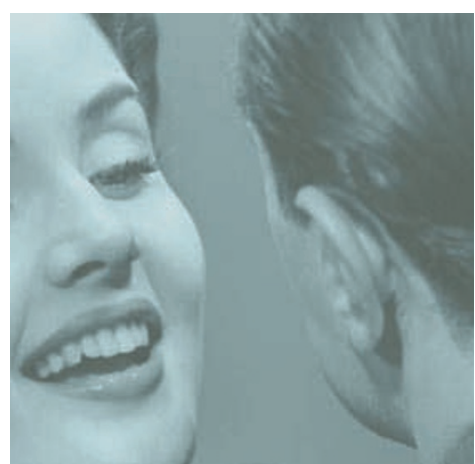

previous studies suggest that many epigenetic marks are likely to change owing to environmental exposures or ageing. The new study - led by the Feinberg, Irizarry and Fallin group - looked at the stability of epigenomic patterns over time. They examined global DNA methylation in samples from 74 participants in the AGES (Age, Gene/Environment Susceptibility) study in Reykjavik, Iceland, who had provided two DNA samples at time points 11 years apart.

The authors used comprehensive high-throughput array-based relative methylation (CHARM) analysis to assess CpG methylation in these samples. From the sample taken at the later time point, they identified 227 regions for which methylation patterns are highly variable among individuals. Feinberg and colleagues then looked at these variably methylated regions (VMRs) in the sample from the earlier time point and found two distinct groups: VMRs that were dynamic over the intervening 11 years and VMRs that remained stable over time.

Do stable VMRs correlate with disease risk? Feinberg and colleagues identified four VMRs that were significantly correlated at both time points with body mass index - a phenotype that is itself correlated with various complex disease phenotypes. Interestingly, some of these VMRs are located at or close to genes that have been previously implicated in obesity.

These findings set the scene for using epigenetic signatures to identify associations between methylation status and disease risk at particular regions of the genome, and potentially to identify new disease-associated genes. An important next step will be to identify more VMRs from larger numbers of individuals from different agegroups and populations.

Louisa Flintoft

ORIGINAL RESEARCH PAPER Feinberg, A. P. et al. Personalized epigenomic signatures that are stable over time and covary with body mass index. Sci. Transl. Med. 2, 49ra67 (2010) 